\title{
Pore-scale insights into transport and mixing in steady- state two-phase flow in porous media
}

DOI:

10.1016/j.jmultiphaseflow.2018.07.006

\section{Document Version}

Accepted author manuscript

Link to publication record in Manchester Research Explorer

\section{Citation for published version (APA):}

Aziz, R., Joekar-Niasar, V., \& Martinez-Ferrer, P. (2018). Pore-scale insights into transport and mixing in steadystate two-phase flow in porous media. International Journal of Multiphase Flow, 109, 51-62.

https://doi.org/10.1016/j.ijmultiphaseflow.2018.07.006

\section{Published in:}

International Journal of Multiphase Flow

\section{Citing this paper}

Please note that where the full-text provided on Manchester Research Explorer is the Author Accepted Manuscript or Proof version this may differ from the final Published version. If citing, it is advised that you check and use the publisher's definitive version.

\section{General rights}

Copyright and moral rights for the publications made accessible in the Research Explorer are retained by the authors and/or other copyright owners and it is a condition of accessing publications that users recognise and abide by the legal requirements associated with these rights.

\section{Takedown policy}

If you believe that this document breaches copyright please refer to the University of Manchester's Takedown Procedures [http://man.ac.uk/04Y6Bo] or contact uml.scholarlycommunications@manchester.ac.uk providing relevant details, so we can investigate your claim.

\section{OPEN ACCESS}




\title{
Pore-scale insights into transport and mixing in steady-state two-phase flow in porous media
}

\author{
Rimsha Aziz ${ }^{\mathrm{a}}$, Vahid Joekar-Niasar ${ }^{\mathrm{a}, *}$, Pedro Martinez-Ferrer ${ }^{\mathrm{b}}$ \\ ${ }^{a}$ School of Chemical Engineering and Analytical Science, University of Manchester, \\ Manchester, M13 9PL, United Kingdom \\ ${ }^{b}$ Centre for Mathematical Modelling and Flow Analysis, Chester Street, Manchester \\ Metropolitan University, Manchester, M1 5GD, United Kingdom
}

\begin{abstract}
Hydrodynamic dispersion and mixing under two-phase flow can be found in many natural, industrial, and engineering processes such as the modified salinity water flooding (MSWF). In MSWF the injected water displaces the formation brine and will interact with the crude oil and rock to improve the oil recovery. We show throughout numerical simulations that access of the injection water to the available pore space is not homogeneous, even in homogeneous porous media, and it is controlled by the saturation topology and pore-scale velocity field.

Under the steady-state two-phase flow in a homogeneous porous medium, the velocity field has a bimodal distribution. The bimodal distribution of pore-scale velocity dictates two different transport time scales spatially distributed over the stagnant and flowing regions at a given saturation topology. These distinctly different transport time scales lead to a non-Fickian transport, which cannot be captured using the conventional advection-dispersion equation.

Using the volume-of-fluid method implemented in the OpenFOAM (ver. 4.0), we simulated pore-scale two-phase flow and the hydrodynamic transport at different saturations. We have investigated the impact of stagnant saturation and the tortuosity of flow pathways on the dispersion coefficient and the mass exchange rate - as the two major parameters controlling transport and mixing - under steady-state two-phase flow. At the Darcy scale,
\end{abstract}

\footnotetext{
* Corresponding author

Email address: vahid.niasar@manchester.ac.uk (Vahid Joekar-Niasar)
} 
different theories such as the mobile-immobile (MIM) theory have been proposed to capture the non-Fickian transport and mixing in two-phase flow through porous media. Based on the simulation results, we assess the validity of the assumptions employed in MIM to define the stagnant saturation and mass exchange rate coefficient. The results of this research provide fresh insights into the potential impact of saturation topology on mixing between the modified salinity water and the formation brine under steady-state flow conditions, which has not been investigated and reported in the literature.

Keywords: Porous media, dispersion, two-phase flow, non-Fickian, pore-scale, mixing

\section{Introduction}

Hydrodynamic dispersion and mixing in two-phase flow through porous 3 media are found in many natural, industrial, and engineering processes such

as contaminant transport in the vadose zone where the infiltrated water carries the contaminants and mixes with the resident water, while air has filled some part of the pore space [37, 4, 23]. Another example is the modified(or low-) salinity water flooding (MSWF) as one of the enhanced oil recovery techniques, in which injection water with a tuned chemical composition is injected into the reservoir filled originally with the formation brine and the crude oil [31, 1, 29, 42]. The performance of MSWF depends on many porescale physio-chemical factors such as crude oil chemistry, formation brine and injection water chemistry, temperature, and rock mineralogy, which have been extensively studied [33, 2, 25]. However, the larger-scale mechanical factors such as transport and mixing of the modified-salinity water have not been extensively studied.

Under single-phase flow conditions, transport strongly depends on the Péclet number (ratio of the advective to the diffusive transport). However, under two-phase conditions, transport and mixing will change strongly with saturation in addition to the Péclet number [11]. While in a homogeneous saturated porous medium, a Fickian transport regime is expected, under twophase flow conditions due to the presence of regions with distinctly different transport time scales, the transport becomes non-Fickian [17, 35, 21], which is saturation- and flow rate-dependent [26, 27].

The void space filled by the carrier fluid (e.g. water) can be decomposed into the backbone, dead-end and disconnected regions [9, 11, 17, 37]. From 
the hydrodynamic point-of-view, they are referred to as flowing (mobile), stagnant (immobile) and non-percolating regions, respectively[17, 27]. The flowing regions contribute to the hydrodynamic solute transport as well as the effective phase permeability, which is captured in the relative permeability curves. The hydrodynamically stagnant regions behave as a sink/source term for the transport process. They do not contribute to flow through a porous medium and consequently they do not contribute to the relative permeability. Finally, the trapped regions contribute neither to transport nor to flow processes. Thus, the total water saturation $\left(S_{w}\right)$ can be decomposed to the flowing saturation $\left(S_{f w}\right)$, stagnant saturation $\left(S_{s w}\right)$, and disconnected saturation $\left(S_{d w}\right)$ such that $S_{w}=S_{f w}+S_{s w}+S_{d w}$. Additionally, while the transport in the flowing region is controlled by the advection, a mass exchange will occur between the flowing and stagnant regions, due to the concentration gradient across the interface between these two regions.

To evaluate the transport parameters, namely dispersion coefficient, effective pore velocity, stagnant saturation, and mass exchange rate coefficient, different models such as the dead-end pore (DEP) [9] and mobile-immobile (MIM) 17] models were proposed. Details of these proposed models can be found in the supplementary information section. Compared to the DEP model, MIM has clear advantages as it captures the non-Fickian behaviour using a non-equilibrium mass exchange between the flowing and stagnant regions [17, 35, 37]. Thus, in several studies the transport and mixing parameters in column-scale two-phase flow experiments were determined using the inverse modelling of the MIM theory [12, 17, 15, 15, 13, 14, 35, 32. Satisfactory results of the inverse modelling were interpreted as an indication of the validity of MIM, although unrealistic values (e.g. the stagnant saturation larger than 1) were reported [32].

Recent direct analysis of micro-scale experiments showed that for a given saturation topology at different flow rates, the same stagnant saturation values were obtained [27]. This is in contradiction with the MIM theory, in which different Péclet numbers would lead to different stagnant saturations, regardless of two-phase flow conditions. Another potential misconception in underlying assumptions employed in MIM is related to the non-equilibrium mass exchange, from inverse modeling a constant mass exchange rate coefficient is obtained, expressed based on the following equation:

$$
S_{s w} \varphi \frac{\partial C_{s w}}{\partial t}=\gamma\left(C_{f w}-C_{s w}\right)
$$


where $S_{s w}, \varphi, C_{f w}$, and $C_{s w}$ are stagnant saturation, porosity, average Darcyscale concentrations of flowing and stagnant regions, respectively. In Equation 1. the rate coefficient, $\gamma$ is assumed to be a constant 17. However, the first direct experimental analysis did not validate this assumption [26].

Former experimental data [27] suggest a non-monotonic trend between the dispersion coefficient and total saturation, as well as the stagnant saturation versus the total saturation. Since the full computational fluid dynamics provides much more detailed information compared to the experiments (such as the detailed velocity field, streamlines and tortuosity), it can be used to evaluate the impact of the velocity field on the delineation of stagnant saturation. Also, change of tortuosity as a function of saturation and its contribution to the transport can be investigated using the CFD simulations. Thus, we employed the direct numerical simulations of hydrodynamic dispersion in two-phase flow through a two-dimensional porous medium using the volume of fluid method implemented in the OpenFOAM software (version 4.0). Furthermore, we explored whether the water relative permeability (as a two-phase flow property) is related to the stagnant saturation.

To achieve these objectives, first, we simulated different steady-state saturation states. Then, at each saturation, hydrodynamic transport at three different injection rates was simulated. Since the numerical grid resolution was much smaller than the smallest pore (15 cells in the smallest pore throat), it enabled us to simulate the detailed sub-pore-scale physics in contrast with the pore-network models where the sub-pore-scale mechanisms have been ignored. We simulated two-phase flow in a two-dimensional domain using the volume-of-fluid method implemented in OpenFOAM. Two-phase flow simulations were performed to establish different steady-state saturations, ranging from 0.34 to 1.0. At each saturation topology, we simulated the advectiondiffusion transport at 3 different injection rates which provided three different macroscopic Péclet numbers of 7, 70, and 700. The Péclet number is defined at the pore scale as well as at the macro scale. At the pore scale, the Péclet number is defined as $P_{e}=\frac{u_{p} \bar{R}}{D_{m}}$, where $u_{p}$ denotes the effective pore velocity, $\bar{R}$ is the mean pore radius, and $D_{m}$ denotes the diffusion coefficient. At the macro scale the Péclet number is defined as $\tilde{P}_{e}=\frac{q L}{D_{m}}$, where $q$ denotes the injection flow rate, $L$ is the macroscopic characteristic length (averaging length), and $D_{m}$ is the diffusion coefficient. For transport simulations, constant concentration and no diffusion boundary conditions were imposed at inlet and outlet, respectively. 
We upscaled the pore-scale simulation results to calculate the relative permeability of the carrier fluid at each steady-state saturation. We also simulated the streamlines at each saturation to obtain the average tortuosity at each saturation. To obtain the dispersion coefficient (as a result of variable flow field), we used an analytical solution to fit the average resident concentration in the flowing region resulted from the pore-scale simulations. The fitting results were excellent with RMSE ranging from $10^{-7}$ to $10^{-5}$. All details covering the theoretical, and computational aspects of the methods, upscaling approach and curve fitting analysis are presented in the following subsections.

\subsection{Volume of Fluid Method}

We simulated immiscible and incompressible two-phase flow in porous media using VoF [40], implemented in OpenFOAM in a solver called interFOAM [43. In the two-phase VoF method, each fluid phase is represented by its volume fraction, $\alpha$. For example, value 1 represents water and value 0 represents oil. In grid cells containing the intermediate values of $\alpha(0<\alpha<1)$, the interface is constructed. The volume fraction $\alpha$ is transported using Equation (2):

$$
\frac{\partial \alpha}{\partial t}+\nabla \cdot(\alpha \mathbf{u})+\nabla \cdot\left(\alpha(1-\alpha) \mathbf{u}_{r}\right)=0
$$

where $\mathbf{u}$ and $\mathbf{u}_{r}$ are referred to as the velocity field and relative velocity between the two fluids $\left(\mathbf{u}_{r}=\mathbf{u}_{1}-\mathbf{u}_{2}\right)$, respectively.

The partial time derivatives in Equation (2) were discretized using the Euler scheme ( $1^{\text {st }}$ order bounded, implicit scheme). The second term in Equation (2) was interpolated using the van Leer flux limiter $\left(2^{\text {nd }}\right.$ order accuracy and bounded) and the third term was linearly interpolated $\left(2^{\text {nd }}\right.$ order accuracy) [43].

The fluids density $(\rho)$ and viscosity $(\mu)$ at the interface were calculated using the weighted averaging represented by $\rho=\rho_{1} \alpha+(1-\alpha) \rho_{2}$ and $\mu=$ $\mu_{1} \alpha+(1-\alpha) \mu_{2} . \rho_{i}$ and $\mu_{i}$ denote density and viscosity of fluid $i$, respectively.

$$
\begin{array}{r}
\frac{\partial \rho \mathbf{u}}{\partial t}+\nabla \cdot(\rho \mathbf{u u})=-\nabla p+\left[\nabla \cdot\left(\mu\left(\nabla \mathbf{u}+\nabla \mathbf{u}^{T}\right)\right)\right]+\mathbf{F}_{s a} \\
\nabla \cdot \mathbf{u}=0
\end{array}
$$


Solving the coupled Navier-Stokes (3a) and continuity equations (3b), pressure $(p)$ and velocity $(\mathbf{u})$ were calculated. $\mathbf{F}_{\mathbf{s a}}$ represents the body force, which includes interfacial forces. The second term on the rhs of Equation (3a) was interpolated using the $2^{\text {nd }}$ order bounded self-filtering central differencing scheme. The second term on the lhs was interpolated using the linear scheme. The pressure field was solved using the bi-conjugate gradient method with a relative tolerance of $10^{-5}$. The velocity field was solved using smooth solver with the relative tolerance of $10^{-10}$. Coupling of pressure and velocity was done using the merged PISO-SIMPLE (PIMPLE) algorithm [40].

The body force, $\mathbf{F}_{s a}$, in Equation (3a) is defined as

$$
\mathbf{F}_{s a}=\rho \mathbf{g} \cdot \mathbf{n}_{\mathbf{z}}+\int_{\Gamma} \sigma \kappa \delta\left(x-x_{s}\right) \hat{\mathbf{n}} d \Gamma\left(x_{s}\right),
$$

where $\Gamma$ is the liquid-liquid interface, and $\delta\left(x-x_{s}\right)$ is the Dirac delta function, $\kappa$ is the curvature of interface, and $\sigma$ is the interfacial tension between the two fluids. Curvature of the interface is $\kappa=-\nabla \cdot\left(\frac{\nabla \alpha}{|\nabla \alpha|}\right)$ and the unit vector $\hat{\mathbf{n}}$ is defined as $\hat{\mathbf{n}}=\frac{\nabla \alpha}{|\nabla \alpha|}$.

The contact angle is defined at the solid boundaries, and is used to determine the volume fraction gradient such that the contact angle satisfies the following equation:

$$
\hat{\mathbf{n}} \cdot \hat{\mathbf{n}}_{\mathbf{s}}=\cos \theta,
$$

where $\hat{\mathbf{n}}$ and $\hat{\mathbf{n}}_{\mathbf{s}}$ are vectors normal to the interface and solid wall, respectively.

The time step $(\delta t)$ in simulations was determined such that Courant number, defined as $C o=\frac{\delta t U}{\delta x}<0.5$. U denotes the magnitude of velocity, $\delta x$ is the cell size. The time steps were around $10^{-6} \mathrm{~s}$.

\subsection{Advection-Diffusion Transport}

Advection-diffusion of a tracer (passive scalar) within the carrier fluid was simulated using Equation (6), where $\mathrm{C}$ is the concentration of the tracer and $D_{m}$ is the molecular diffusion coefficient, which is typically $10^{-9} \mathrm{~m}^{2} / \mathrm{s}[10]$.

$$
\frac{\partial C}{\partial t}+\mathbf{u} \cdot \nabla C-\nabla \cdot\left(D_{m} \nabla C\right)=0
$$

The steady-state velocity field at each saturation was used to simulate transient advection-diffusion in the domain using scalar TransportFoam solver. 
Simulated concentration field at each time scale was averaged to obtained the Darcy-scale resident concentration curves. The resulted concentration-time curves were used to estimate the hydrodynamic dispersion and mass exchange rate of the domain.

The advective term was interpolated using upwind ( $1^{\text {st }}$ order, explicit) method and the diffusive term was discretized using Gaussian linear corrected scheme ( $2^{\text {nd }}$ order). The time derivative (for concentration) was discretized using the Euler $\left(1^{\text {st }}\right.$ order) scheme. The transport matrix was solved using smoothSolver due to the sharp gradient of concentration between stagnant and flowing saturation with the relative tolerance of $10^{-16}$.

\subsection{Numerical domain, boundary, and initial conditions}

The numerical domain had a square 2D geometry. The domain has been shown in Figure S1 in the Supporting Information. Since at each direction there were more than 30 pores and the grains were homogeneously distributed, the domain is definitely larger than Representative Elementary Area (REA) [24]. Top and bottom boundaries shared no-flow conditions. The right boundary was at constant pressure with zero gradient of solute concentration normal to the boundary. The left boundary was at constant inlet injection velocity and constant dimensionless solute concentration. Initially, the domain was fully saturated with the wetting phase $(\alpha=0)$ with a contact angle of 140 degrees (measured through the non-wetting phase). The non-wetting phase (i.e. water in an oil-wet medium) was injected at a constant rate.

The domain was meshed using the OpenFOAM utility snappyHexMesh. This tool allowed the refinement of grain edges to ensure a high-quality mesh in narrower pore throats. We performed the grid convergence analysis in a sub-section of the original domain (Figure S2 in the Supporting Information). Based on the grid convergence analysis, we chose a mesh with 3.6 million cells, which allows at least 15 cells in the narrowest pore throat. Different steadystate two-phase saturation topologies were established (ranged between 0.341) to be used for transport simulations.

\subsection{Material properties}

The density and viscosity of $998\left(\mathrm{kgm}^{-3}\right)$ and $10^{-3}\left(\mathrm{kgm}^{-1} \mathrm{~s}^{-1}\right)$, respectively, were assigned to the advancing fluid, which carried the solute. The receding fluid had the density and viscosity of $844\left(\mathrm{kgm}^{-3}\right)$ and $1.910 \times 10^{-2}$ $\left(\mathrm{kgm}^{-1} \mathrm{~s}^{-1}\right)$, respectively. The interfacial tension, $\sigma$, was $0.07\left(\mathrm{kgs}^{-2}\right)$ and 
the solid surface had affinity to the receding phase with a contact angle of 140 degrees, measured through the advancing phase. The diffusion coefficient $\left(D_{m}\right)$ of $10^{-9}\left(\mathrm{~m}^{2} \mathrm{~s}^{-1}\right)$ was assumed [10]. The concentration reported in the study is dimensionless, therefore the maximum value is 1 and minimum is 0 .

\subsection{Upscaling the simulation results}

The MIM theory is a Darcy-scale model, while the numerical results are defined in grid cells much smaller than a pore. To evaluate the parameters at the Darcy scale, it is essential to upscale (average) the sub-pore-scale entities. We averaged the grid-based results to estimate the transport parameters at the Representative Elementary Area (REA) scale, which represents the Darcy scale.

\subsubsection{Dispersion coefficient of the flowing network}

Combined effects of heterogeneous velocity distribution and tortuosity lead to the hydrodynamic dispersion. It is known that the hydrodynamic dispersion is strongly flow-rate dependent 38 . To obtain the hydrodynamic dispersion either the method of moments [6] or fitting the data to the analytical solutions [3] can be used. For the Dirichlet boundary condition of our simulations, the one-dimensional analytical solution developed by Ogata and Banks was used [34]. This equation is originally developed for the singlephase flow in semi-infinite domain. There is a complicated analytical solution for finite domain as discussed in the literature [16]. However, breakthrough analysis shown in the literature illustrate that the results of the complicated analytical solution for a finite domain will be identical to the results of the Ogata-Bank equation for Péclet numbers larger than 5 [16]. Therefore, given the simplicity and better convergence of the Ogata-Banks equation for curve fitting and since the macroscopic Péclet number is much larger than 5 in our simulations, we performed the curve fitting using the Ogata-Banks equation. Under two-phase flow, this equation can only be used to estimate the dispersion coefficient of the flowing network, as the flowing network in a fixed saturation topology obeys the Fickian transport [17, 11, 26]. Note that due to the injection of the solute from the whole cross section and no-flow side boundaries, the lateral dispersion was negligible. 


$$
\begin{aligned}
\left\langle C_{f}(t)\right\rangle & =\frac{1}{L} \int C_{f}(x, t) d x \\
& =0.5 \frac{1}{L}\left[\left(\left(\frac{2 \sqrt{D t}}{\sqrt{\pi}}\left(e^{-\Lambda_{2}^{2}}-e^{-\Lambda_{1}^{2}}\right)\right)+(t \bar{u}) \operatorname{erfc}\left[-\Lambda_{2}\right]\right.\right.
\end{aligned}
$$$$
\left.+(L-t \bar{u}) \operatorname{erfc}\left[\Lambda_{1}\right]\right)+\left(\frac { D } { \overline { u } } \left(\operatorname{erf}\left[\Lambda_{2}\right]+\operatorname{erf}\left[\Lambda_{1}\right]-\operatorname{erfc}\left[\Lambda_{2}\right]\right.\right.
$$$$
\left.\left.\left.+e^{\frac{L \bar{u}}{D}} \operatorname{erfc}\left[\Lambda_{3}\right]\right)\right)\right], x
$$

$$
\begin{gathered}
\in \Omega_{S_{f}} \\
\Lambda_{1}=\frac{L-t \bar{u}}{2 \sqrt{D t}}
\end{gathered}
$$

$$
\Lambda_{2}=\frac{t \bar{u}}{2 \sqrt{D} t} ;
$$

$$
\Lambda_{3}=\frac{L+t \bar{u}}{2 \sqrt{D t}}
$$

This analytical equation captures the Fickian spatio-temporal evolution of the resident concentration and in order to avoid the uncertainty of the fitting results in early times, we used the analytical solution integrated over the domain $(L)$. Since the resulted equation can only capture the Fickian transport, it is only applicable to the transport in flowing regions. Thus, the average resident concentration in the flowing regions, $\left\langle C_{f}(t)\right\rangle$, was calculated from the simulation results using the cell area-weighted averaging. $\left\langle C_{f}(t)\right\rangle=\Sigma\left(C_{i}(t) A_{i}\right) / \Sigma A_{i}, A_{i} \in \Omega_{f} . C_{i}$ is the concentration in cell $i$ with the surface area of $A_{i} . \Omega_{f}$ represents the domain of flowing regions. By fitting the $\left\langle C_{f}(t)\right\rangle$ using Equation (7), effective pore velocity, $\bar{u}$, and dispersion coefficient, $D$, were obtained.

In all cases, the curve fitting results were excellent with RMSE ranging from $10^{-7}-10^{-5}$. Two examples of the curve fitting at two different saturations at the Pećlet number of 700 are shown in Figure S3 of the Supporting Information.

\subsubsection{Relative permeability of the carrier fluid}

Since the stagnant regions do not contribute to flow, the relative permeability curves capture the net contribution of the flowing regions. Hence, 
relative permeability curves potentially carry important information about the stagnant saturations.

The relative permeability, $k_{r}$, is defined as the ratio of the effective permeability at a given saturation $k_{e f f}(S)$ to that of a fully-saturated network, as shown in Equation (8). The effective permeability was calculated using Darcy's law, as follows

$$
k_{r}(S)=\frac{k_{\text {eff }}(S)}{k_{\text {eff }}^{*}}=\frac{Q(S) A^{*}\left(P_{\text {in }}-P_{\text {out }}\right)}{Q^{*} A(S)\left(P_{\text {in }}^{*}-P_{\text {out }}^{*}\right)},
$$

where $*$ denotes the values calculated/used under the fully-saturated conditions and $A$ refers to the cross-sectional area at the inlet filled with the invading fluid. Inlet and outlet pressures are denoted by $P_{\text {in }}$ and $P_{\text {out }}$, respectively. Total flow rate is illustrated by $Q$. Note that since the relative permeability was calculated under steady-state conditions, no dynamic effect was included.

\subsubsection{Tortuosity}

Tortuosity is a measure of the sinuosity of flowing network pathways. To calculate the tortuosity at steady-state saturations, the steady-state velocity field within the carrier (invading) fluid was used to construct the streamlines (following the streamline equation $\frac{d x}{u_{x}}=\frac{d y}{u_{y}}$ in a $2 \mathrm{D}$ velocity field $u=\left(u_{x}, u_{y}\right)$ ). The total number of streamlines $(N=5000)$ was fixed for the fully saturated case. Based on the value of $N$ for the fully saturated case, the density of streamlines was calculated. Then using the same density, number of streamlines was scaled for each saturation case. The tortuosity at each saturation was calculated as the average length of all streamlines divided by the domain length. Sensitivity analysis was performed by increasing the density of streamlines in the inlet, which did not show any impact on the results.

\section{Results and discussion}

Figure 1 visualises simulation results at 4 different saturations. This figure matrix shows the Péclet number fields, stagnant and flowing regions, stagnant and total saturation, streamlines and tortuosity, and transient concentration fields at 4 different saturation topologies. Rows 1 and 2 show the amount of total and stagnant saturation, respectively. The method to estimate the stagnant saturation will be explained later in detail. Row 3 shows the spatial distribution of the pore-scale Péclet number. It is clearly visible that the 

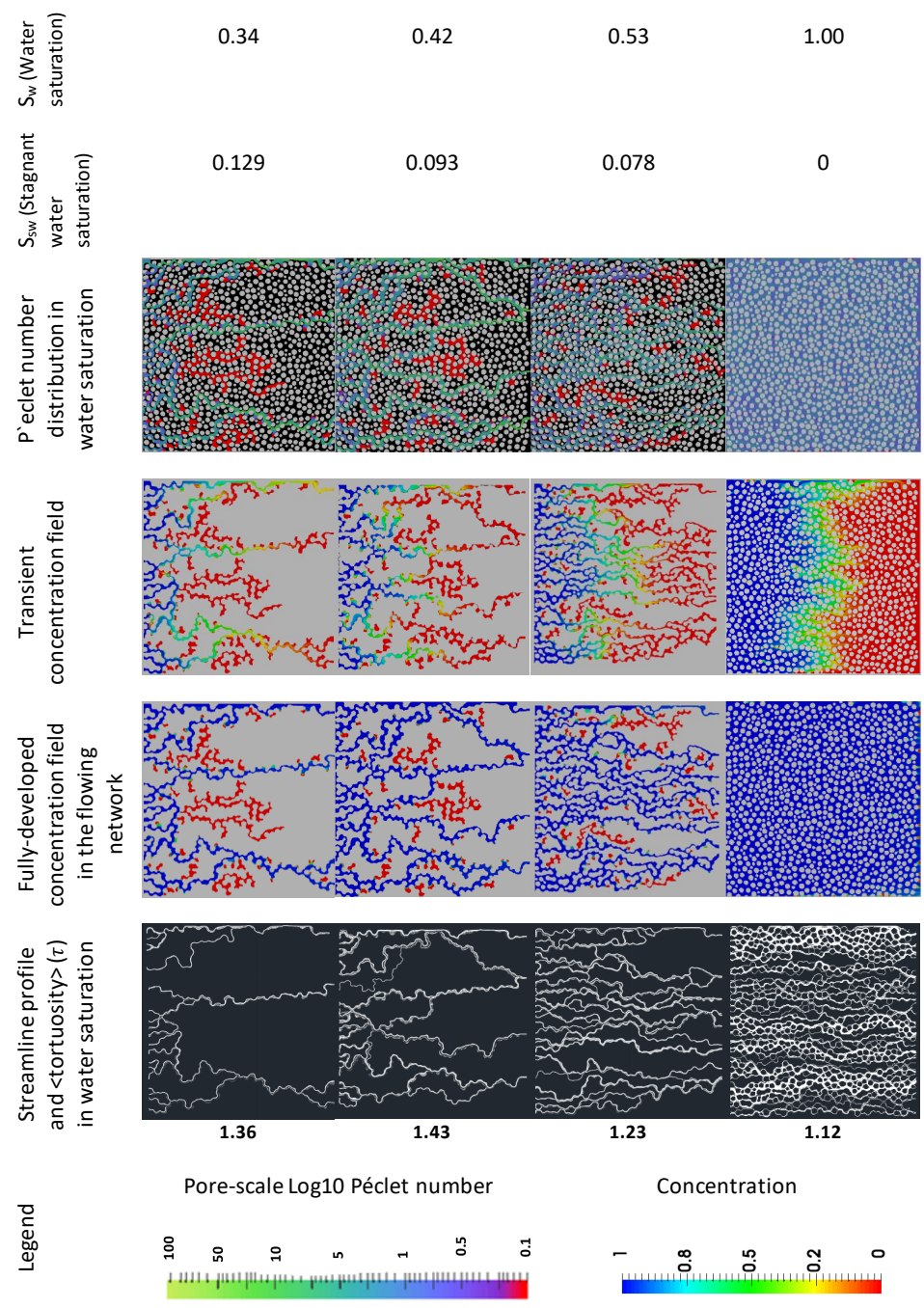

Figure 1: Simulation results for 4 different saturation topologies. Row 1: values of the total water saturation $\left(S_{w}\right)$. Row 2: stagnant water saturation $\left(S_{s w}\right)$. Row 3: topology of the two fluid phases (black: receding fluid) and the local Péclet number distribution in water phase shown by the logarithmic-scale colour coding. Row 4: concentration fields at the total resident concentration of 0.5 and the macro-scale Péclet number of 700 . Row 5: the concentration profile at the moment of the full development of the concentration field in the flowing network at the Péclet number of 700. Stagnant and flowing regions are shown in red and blue, respectively. Row 6: streamlines within the water phase. The values under the visualisation of streamlines show the corresponding average tortuosity values. 
pore-scale Péclet number (and the velocity field) spatially varies by orders of magnitude in steady-state two-phase flow. Row 4 shows the snapshots of concentration field when the total resident concentration is 0.5 . The figures qualitatively show that under two-phase flow conditions the spatial distribution of concentration is more heterogeneous compared to the single-phase flow condition. The stagnant and flowing regions are shown in the $5^{\text {th }}$ row. The dead-end pores, which do not contribute to the flow and have null concentration, are diffusion-controlled and are shown in red and the flowing regions are shown in blue. Finally, the row 6 shows the streamlines at different saturations and the average tortuosity has been shown for each saturation.

\subsection{Stagnant saturation as a hydrodynamic entity}

In previous macroscopic experimental studies [32, 35, 19, 5], the stagnant saturation was estimated by fitting the resident or breakthrough concentration time series to the MIM model. Note that in the MIM model if the flow rate is very small, the concentration profile does not show significant non-Fickian behaviour. Thus, the MIM fitting results will indicate a very small or zero stagnant saturation. However, by increasing the flow rate at the same steady-state saturation topology, the difference between the transport time scales in the flowing and stagnant regions will increase. This can be interpreted as a flaw, since as long as the saturation topology does not change, the stagnant saturation should not change. This has been verified in a recent experiment[27]. That is potentially the cause of the significant data scattering in the relation between the stagnant saturation and the total saturation (see Fig.5 in the reference [26]). Additionally, it has been proven experimentally that for a given saturation, different saturation topologies are possible [18], which can lead to different stagnant saturation values.

To estimate the stagnant saturation at a given saturation topology, we have simulated the velocity field under the steady-state two-phase flow. The probability distributions of the pore-scale Péclet numbers (for the macro scale Péclet number of 700) at 6 different saturation topologies are shown in Figure $2 a$ and the corresponding cumulative probability distributions have been shown in Figure $2 \mathrm{~b}$. These distributions are fundamentally different as they show a single-mode distribution under single-phase flow (Figure 2 ab) and bimodal distributions in the steady-state two-phase flow (two peaks in Figure 2a. The second mode with very small Péclet numbers represents the hydrodynamically stagnant regions (the lhs peak on Figure 2a). Taking the saturation of 0.34 as an example, there are two large peaks, which 


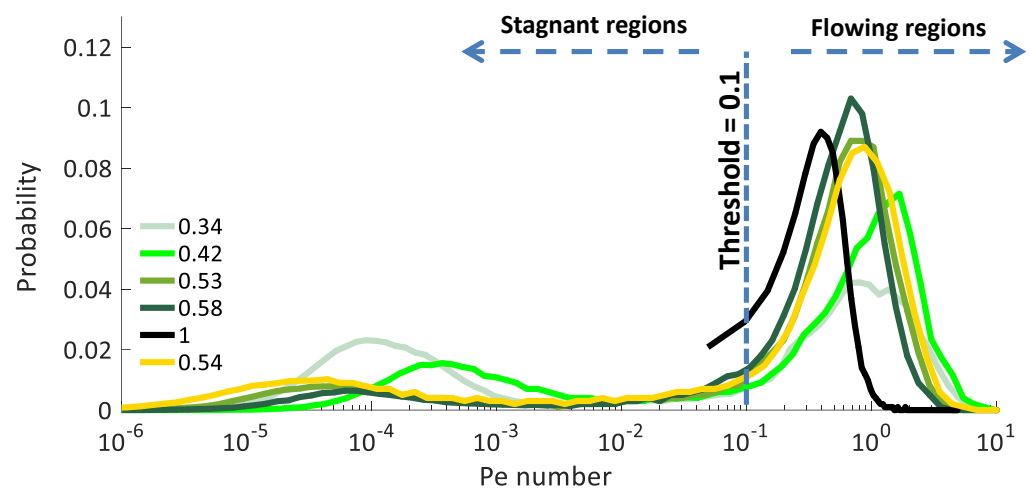

(a)

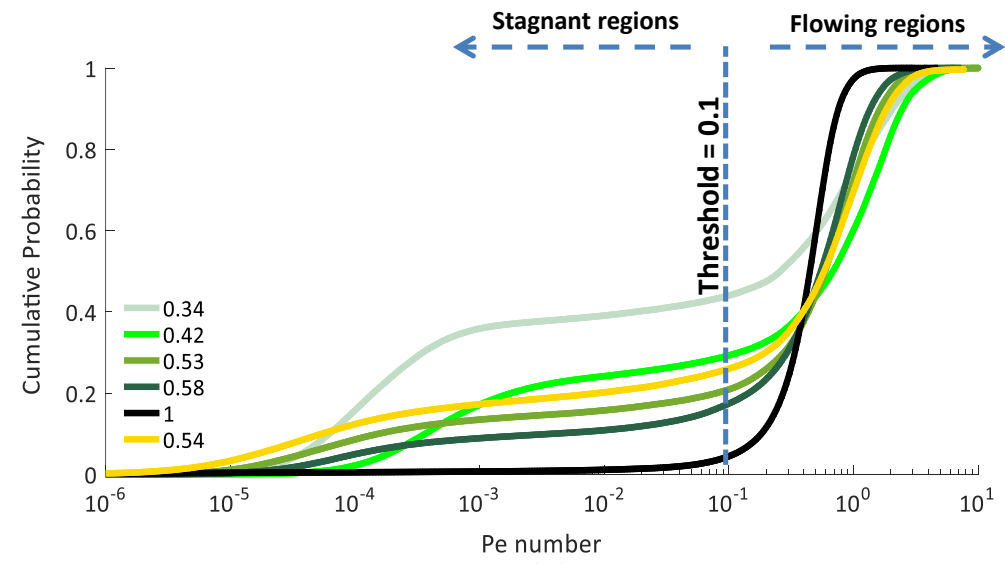

(b)

Figure 2: a) Probability distribution of the pore-scale Péclet number in the numerical cells. There is a clear shift from single-mode distribution in a fully-saturated porous media to a bimodal distribution under two-phase flow conditions. The probability distribution illustrates the stagnant (the lhs peak) and flowing regions (the rhs peak). The Péclet number ranging from 0.01 to 0.1 shows a transition zone. We have selected the $P e=0.1$ as the threshold value that differentiates the flowing and stagnant regions. b) Cumulative probability distribution of results shown in figure (a). 

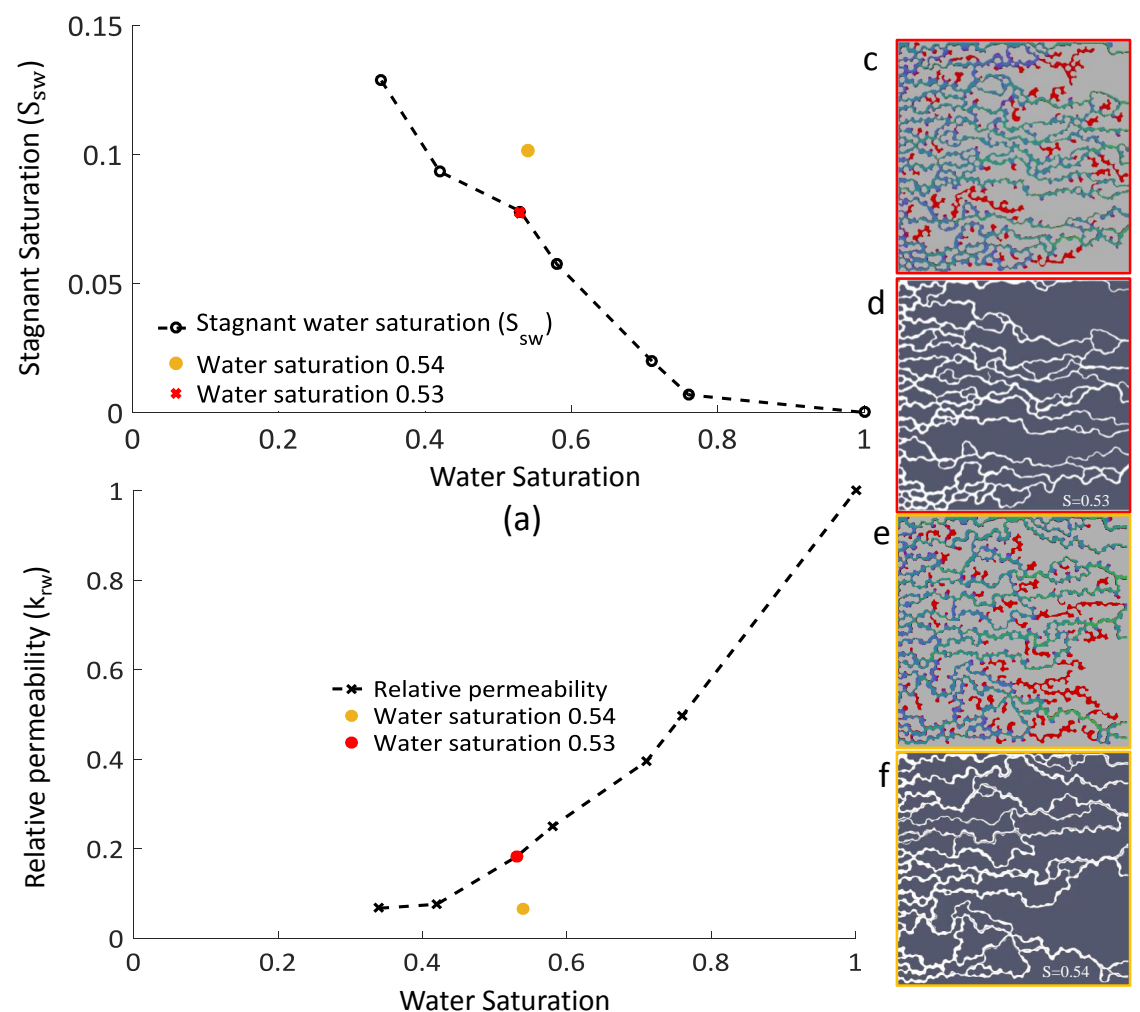

(b)

Figure 3: a) Stagnant saturation against the total saturation, which is identical for three different injecting rates representing macro-scale $P e=7,70,700$ for the same saturation topologies, b) Water relative permeability (which is the advancing fluid) versus the total saturation. The relative permeability and stagnant saturation at two different saturations of 0.53 and 0.54 are shown in red and orange circles, respectively. c and e) Fluids topologies for the saturations of 0.53 and 0.54 , respectively, $\mathrm{d}$ and f) streamlines of saturations 0.53 and 0.54 , respectively. 
clearly show dual transport time scales. The bimodal velocity field indicates that some parts of the pore space filled with water (belonging to the flowing domain $\Omega_{f w}$ ) are highly advective and some other parts belonging to the stagnant regions $\left(\Omega_{s w}\right)$ are diffusion-controlled, which leads to an upscaled non-Fickian transport process. Single-phase transport experimental and computational data show that the Péclet number of 0.01 to 0.1 is the threshold above which the advective transport starts to be pronounced [3]. Thus, we chose 0.1 as the threshold to differentiate the flowing and stagnant regions.

Note that under the steady-state two-phase flow, the difference between transport time scales in these two regions increases with the increase of flow rate; as the stagnant regions are always diffusion controlled and the transport time scale in the flowing regions becomes smaller with the increase of flow rate.

The relation between the stagnant saturation and total saturation for three different injection rates is shown in Figure 3 a. These results are in qualitative agreement with former studies, which showed the stagnant saturation increased with the decrease of the carrier fluid's saturation [21, 15, 13, 19]. However, unlike the experimental data by [26, 27] which showed a nonmonotonic trend, no non-monotonic trend is observed in the numerical simulations. This can be due to the fact that in the micromodel experiments [26, 27], the smallest percolating water saturation was 0.2. However, in our simulations, the smallest percolating saturation is about 0.34 . This significant difference between the smallest possible percolating saturations may have potentially led to the difference in trends.

Since the stagnant saturation does not contribute to flow, its signature should be visible in two-phase flow relative permeability curves. To examine the correlation between the stagnant saturation and relative permeability, the steady-state water relative permeability-saturation curve has been shown in Figure $3 \mathrm{~b}$. Also, an additional case with the saturation of 0.54 was simulated. Although saturations 0.53 and 0.54 are very similar, their saturation topologies are different. Hence, the relative permeabilities of saturations 0.53 and 0.54 are significantly different, as shown in red and orange circles in Figure 3 b, respectively. Also, their stagnant saturations and the streamlines are significantly different (Figure 3c-f). The water relative permeability of the saturation 0.54 is smaller than the water saturation of the case 0.53. Accordingly the stagnant saturation is larger for the case of saturation $0.54(0.102$ versus 0.078, Figure 3a). The topology and streamlines of both cases are shown in Figures 3 c-f. As shown in those Figures, the saturation topology is 
very important and this indicates the reason for non-unique relation between the stagnant saturation and total saturation which has been shown in the literature [26].

Our results suggest that the two-phase transport modelling can be integrated with the two-phase flow modelling by establishment of a relation between the stagnant saturation and the water relative permeability. This is an important concept in the modelling as currently the two-phase flow simulations and the solute transport simulations do not communicate with each other through a physically-consistent concept; models such as MIM do not capture any parts of two-phase flow in their formulations. Further research is required to develop full understanding of the correlation between the relative permeability and stagnant saturation and direct estimation of the stagnant saturation from the two-phase flow data. 


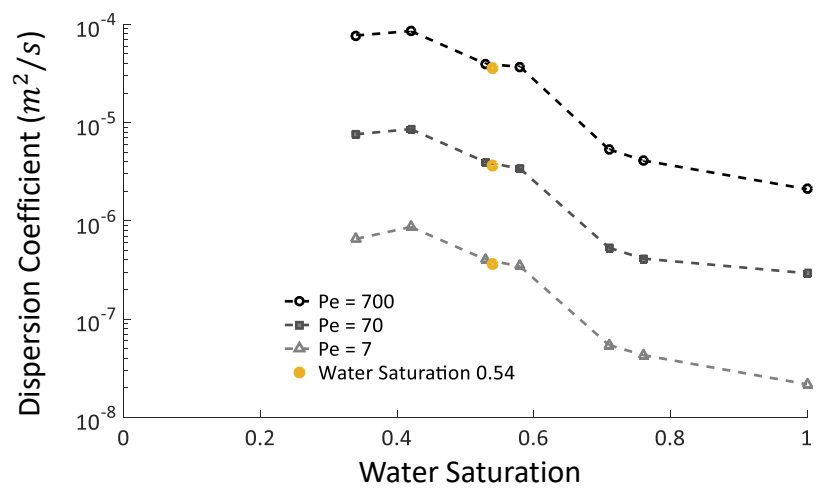

(a)

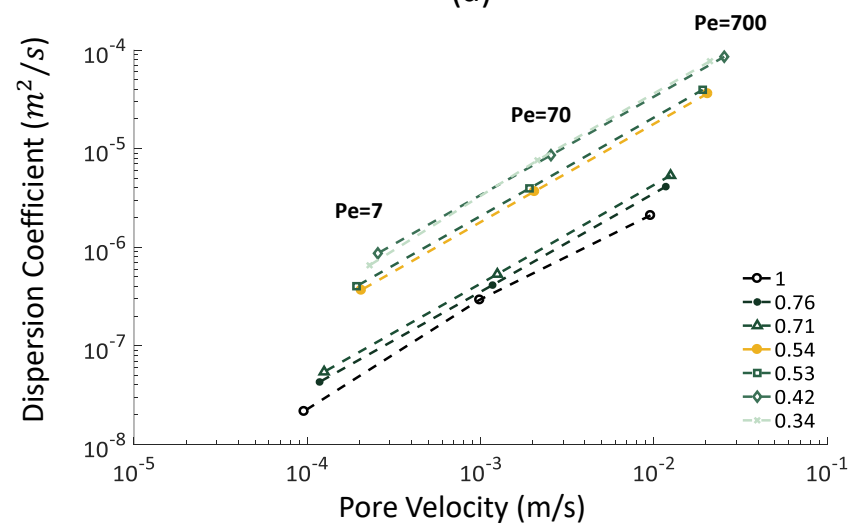

(b)

Figure 4: a) Dispersion coefficient (D) of the flowing network plotted against saturation for the macro scale Péclet numbers of 700,70 and 7 . b) Dispersion coefficient (D) for flowing network plotted against the pore velocity $(\bar{u})$ at different saturations. The yellow circles in figures a and b indicate the case of saturation of 0.54 . 
By decomposing the water saturation to the flowing and stagnant regions, the transport in the flowing regions follows the Fickian transport. Thus, the dispersion coefficient and pore velocity can be obtained by the conventional curve fitting to the advection-dispersion equation, as discussed in the Supplementary Information. Figure 4 a shows that under fixed flow boundary conditions, two-phase dispersion coefficient can be orders of magnitude larger than the dispersion coefficient in a fully-saturated system. Although all former studies indicate that the dispersion coefficient under two-phase flow conditions is larger than that under single-phase flow conditions, there is no clear agreement in the literature on factors controlling the dispersion coefficient. Some former studies reported that dispersion coefficient decreases as stagnant saturation increases [15, 22, while some other studies speculated that dispersion coefficient increases under two-phase conditions due to the presence of stagnant regions [14, 30, 20]. There are studies which suggest a non-monotonic relationship between the dispersion coefficient and saturation [41, 26, 27] and conjectured that the non-monotonicity in the stagnant saturation caused the non-monotonicity in the dispersion coefficient. This can also be seen in Figure1, row 4, where the concentration fields at different saturations are shown under the transient condition. The longitudinal dispersion varies significantly between saturation 1 and 0.34 . Stagnant saturation is monotonically increasing with decrease of the carrier fluid's saturation. However, there is a slight non-monotonicity in the dispersion coefficientsaturation relation (Figure 4a).

Increase of the dispersion coefficient of the flowing region under two-phase conditions compared to the single-phase case is due to increase of the tortuosity as well as the increase in the stagnant saturation. Tortuosity of the water-filled area will change significantly as a function of saturation topology as shown in Figure 5 and Figure 1, row 6. In contrast to the stagnant saturation, which is monotonically changing with saturation in our simulations, the tortuosity is a non-monotonic function of saturation and it may explain the slight non-monotonicity in the dispersion coefficient-saturation relation. With decrease of the flowing saturation, not only the tortuosity increased (Figure 5 ) but also the available flowing path (i.e. relative permeability) decreases, which leads to a large dispersion coefficient of the flowing region. This is in agreement with the work of Sahimi et al. [37. The flowing pathways are shown with snapshot of streamlines of water saturation in Figure 1. row 6 . The decrease of flowing pathways can clearly be seen in Figure 1. row 6 between water saturation 0.42 and 0.34 . If only the number of flowing 


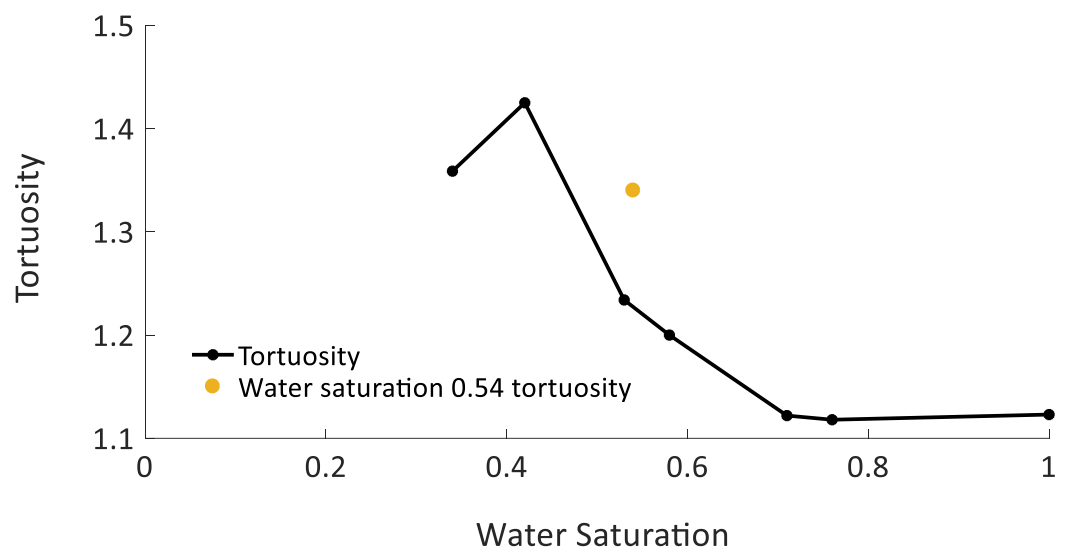

Figure 5: Variation of the average tortuosity with total saturation. The solid circle shows the tortuosity for the case of saturation of 0.54 , respectively.

pathways affects dispersion coefficient, we would expect an increase of the dispersion coefficient between water saturation of 0.42 and 0.32 . However we observe a decrease in dispersion coefficient. This is because even though the number of pathways are decreasing, the tortuosity of water pathways are also decreasing, which results in a small decrease in dispersion coefficient in Figure 4 . Note that there is not necessarily a relation between the relative permeability and tortuosity. As shown in Figure 5, for the saturation range of 0.7 to 1 , the tortuosity is almost constant while the relative permeability (Figure 3) drops significantly in the same range of saturation. That indicates that some of the main flow paths have been completely lost by the decrease of saturation from 1 to 0.7 , while their loss does not impact the tortuosity of the remaining flow paths. The trend of tortuosity is our simulation results is fundamentally different from the tortuosity-saturation relation proposed by Burdine [8]. Burdine proposed a linear relation between tortuosity and saturation following the same trend as relative permeability, which does not seem to be correct.

The dispersion coefficient has always been reported to have a linear relation with the pore velocity, regardless of saturation in literature [26, 32, 12, 15, 22, 30, 20, 38, 28, 44, 39, 36, 7]. As shown in Figure 4b, we have got similar trends between the hydrodynamic dispersion and the effective pore velocity at each given saturation. However, the relation is saturation-dependent and 
non-universal.

\subsection{Mass exchange rate coefficient}

As discussed earlier, during the transport process, the injected fluid mixes with the resident fluid. This mixing in two-phase flow is a function of saturation as the stagnant saturation varies significantly with the total saturation. The mass exchange rate coefficient $(\gamma)$ as defined in Equation (1) is an important parameter that controls the mixing between the stagnant and flowing regions [17.

Using the detailed numerical results, we evaluated the functionality of $\gamma$ at different saturations. Knowing the temporal evolution of the resident concentration in the flowing and stagnant regions and the amount of the stagnant saturation, we calculated the mass exchange rate coefficient $\gamma$ versus time using Equation 1. The results are shown in the inset Figure 6c. for four different saturations. Figures 6 a and b show the total residence concentration (C) (blue dashed curve), average resident concentration in the stagnant regions, $C_{s w}$ (yellow dashed curve) and flowing regions, $C_{f w}$ (red dashed curve). The concentrations have been plotted against the dimensionless time (defined as total fluid volume injected by a given time divided by total volume of the carrier fluid in the domain, $\left.\bar{t}=\frac{Q_{w} t}{V_{p} S_{w}}\right)$ in a logarithmic scale. The plots show that the concentration in the flowing regions reach the inlet concentration in a short time (in the order of 2 pore volumes) and follows a Fickian regime. However, the concentration in stagnant regions (yellow dashed curves in Figures $6 a$ and b) build up very slowly that leads to a long tailing (non-Fickian behaviour) in the total concentration profile (dashed blue curve in figure 6a-b). This slow development of concentration in stagnant regions, which is controlled by diffusion, is due to the exchange between the flowing and stagnant region interface. Exchange rate coefficients $(\gamma)$ calculated for four different saturations, are shown in 6c for the Péclet number 700 .

Our results clearly shows that $\gamma$ versus time is not constant, opposed to the assumption of MIM. $\gamma$ exhibits two stages with time. Stage 1, before the full development of the concentration field in the flowing network: $\gamma$ increases as the mixing line between the flowing and stagnant regions is increasing. Stage 2, after the full development of the concentration field in the flowing saturation, $\gamma$ decreases gradually due to the depleting concentration gradient across the interface between the flowing and stagnant regions. Consequently, 

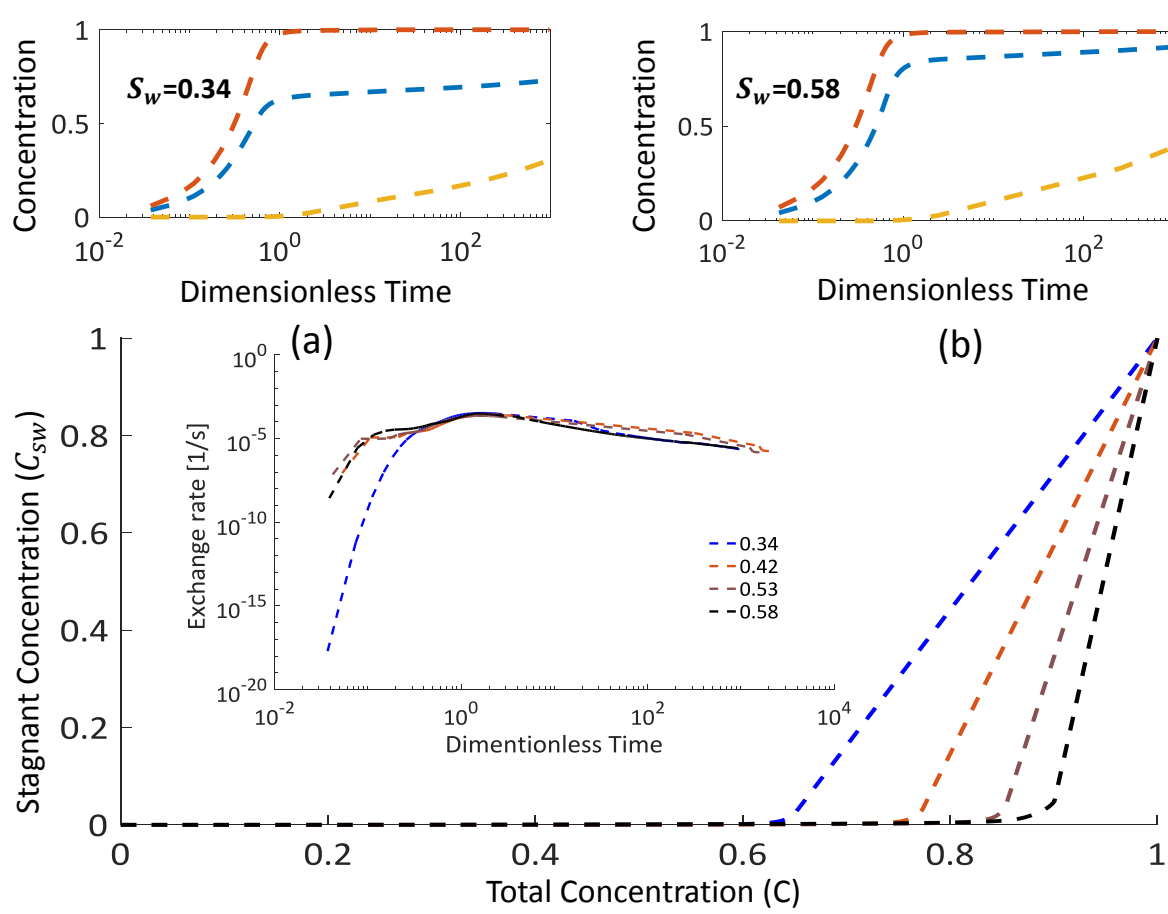

(c)

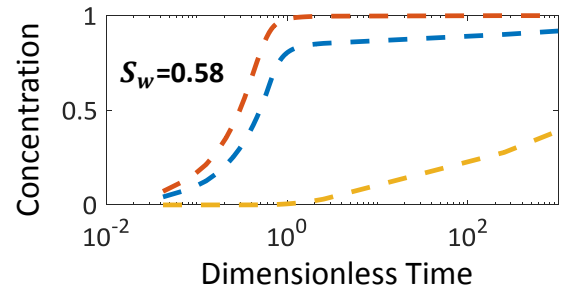

(b) 1 
the ever-decreasing $\gamma$ prolongs the mixing that indicates the overestimation of mass exchange rate in the MIM model.

The variable rate coefficient was also reported in the experimental results [26], although the experiments did not capture the long-term behaviour of the mass exchange due to the short duration of the experiments. Our results support the experimental study and clearly shows that exchange rate varies with time and saturation.

Our results imply that the existing mass exchange models do not capture the long term behaviour of mixing in two-phase flow. This understanding has direct implications for modified salinity water flooding (MSWF) technique. While the injected water can replace the formation brine resident in the flowing regions at a short time scale, the stagnant regions will remain filled by the formation brine for a much longer time. The high salinity water in the stagnant regions will be gradually diluted with the injected modified salinity water, which is controlled by the mass exchange rate. As our results show, the mass exchange rate coefficient decreases with time, which can possibly deplete the MSWF performance at much longer time scales. Moreover, since the amount of stagnant saturation varies with total saturation, it is speculated that the performance of wettability alteration would significantly change at different saturations.

\section{Conclusions}

Understanding and modelling of hydrodynamic transport and mixing in two-phase flow through porous media are important for many natural, industrial and engineering applications, such as modified salinity water flooding for enhanced oil recovery. Our results and former studies in the literature show that under steady-state two-phase conditions (even in homogeneous porous media), the void space filled by the carrier fluid (e.g. water) will have two hydrodynamically different regions; stagnant regions and flowing regions. These two regions have two different transport time scales that can lead to a non-Fickian transport regime. The stagnant regions are diffusion controlled, while the flowing regions are advection-controlled. Thus, increase of the injection rate will make the transport in the flowing regions faster. Consequently, the non-Fickian behaviour becomes more pronounced with the increase of injection rate.

Moreover, the stagnant saturation varies with total saturation, which means the non-Fickian behaviour strongly depends on saturation. 
The existing models for transport under two-phase flow conditions such as the Mobile-Immobile (MIM) model do not incorporate any two-phase flow property in their formulations and make some assumptions and their validity has not been addressed in the literature. Our results show that stagnant saturation is inversely proportional to the relative permeability. This implies that a relation should be established to estimate the stagnant saturation from the relative permeability, which allows coupling two-phase flow and transport in a physically-consistent way. This is absolutely absent in the MIM or other transport models.

Moreover, our results challenge the assumptions related to the stagnant saturation and mass exchange rate coefficient incorporated in MIM. MIM formulations allow having different stagnant saturations for a given saturation topology at two different Pećlet numbers, which contradicts the hydrodynamic definition of the stagnant saturation. Experimental data[27] and our numerical simulations indicate that as long as the saturation topology does not change, the stagnant saturation should not change.

Additionally, MIM assumes a linear non-equilibrium mass exchange between the flowing and stagnant regions with a constant mass exchange rate coefficient. Our results did not support the MIM's assumptions as the rate coefficients were variable with time. Two stages were identified in rate coefficients. In the early times, the rate coefficient increased with time until the concentration field in the flowing network was fully developed. Then, it gradually decreased with time. The results showed that the mass exchange rate coefficient in stage 1 was highly dependent on the stagnant saturation's geometrical and topological distribution. But, after full development of the concentration fields in the flowing regions, the trend mass transfer rate coefficients at different saturations were not very different.

While our results provide fresh insights into the potential mixing and transport processes which are applicable to the modified salinity water flooding, we have not yet incorporated the wettability alteration or other induced mechanisms in our model. Transport and mixing under dynamic two-phase flow are integral aspects of modified salinity water flooding, which will be investigated in future publications.

\section{Acknowledgments}

The authors would like to acknowledge the UK Engineering and Physical Sciences Research Council (EPSRC) for funding the PhD studentship 
of Miss Rimsha Aziz. They like to acknowledge the assistance given by IT Services and the use of the Computational Shared Facility at The University of Manchester.

\section{Author Information}

\section{Corresponding Author}

*Telephone: +44-16130-64867. Email: vahid.niasar@manchester.ac.uk.

Author Contributions

Rimsha Aziz performed the numerical simulations, and data analysis. Vahid Joekar-Niasar defined and led the research project and objectives. Pedro J.Martinez Ferrer supported troubleshooting of OpenFOAM simulations. The manuscript was written through the contribution of Vahid Joekar-Niasar, Rimsha Aziz and Pedro J.Martinez Ferrer. All authors have given approval to the final version of the manuscript.

\section{Data availability statement}

The data will be made available upon publication on the IMPRES group website (http://personalpages.manchester.ac.uk/staff/vahid.niasar/default.htm).

\section{Supplementary Information}

The supporting document presents the numerical domain, the convergence analysis for determining the grid size and the curve fitting results. 


\section{References}

[1] Amirian, T., Haghighi, M., Mostaghimi, P., 2017. Pore scale visualization of low salinity water flooding as an enhanced oil recovery method. Energy \& Fuels 31, 13133-13143.

[2] Austad, T., Rezaeidoust, A., Puntervold, T., 2010. Chemical mechanism of low salinity water flooding in sandstone reservoirs, in: SPE Improved Oil Recovery Symposium, 24-28 April, Tulsa, Oklahoma, USA, Society of Petroleum Engineers. Society of Petroleum Engineers.

[3] Babaei, M., Joekar-Niasar, V., 2016. A transport phase diagram for pore-level correlated porous media. Advances in Water Resources 92, 2329. URL: http://www.sciencedirect.com/science/article/pii/ S0309170816300781, doi:http://dx.doi.org/10.1016/j.advwatres. 2016.03.014.

[4] Birkholzer, J., Tsang, C.F., 1997. Solute channeling in unsaturated heterogeneous porous media. Water Resources Research 33, 22212238. URL: http://dx.doi.org/10.1029/97WR01209, doi:10.1029/ 97WR01209.

[5] Bond, W.J., Wierenga, P.J., 1990. Immobile water during solute transport in unsaturated sand columns. Water Resources Research 26, 2475-2481. URL: http://dx.doi.org/10.1029/WR026i010p02475, doi:10.1029/WR026i010p02475.

[6] Bosma, W.J.P., van der Zee, S.E.A.T.M., 1995. Dispersion of a continuously injected, nonlinearly adsorbing solute in chemically or physically heterogeneous porous formations. Journal of Contaminant Hydrology 18, 181-198. URL: http://www.sciencedirect.com/science/ article/pii/016977229500005G, doi/http://dx.doi.org/10.1016/ 0169-7722(95)00005-G.

[7] Brigham, W.E., Reed, P.W., Dew, J.N., 1961. Experiments on mixing during miscible displacement in porous media. SPE Journal 1, 1-8. doi:10.2118/1430-G.

[8] Burdine, N., 1953. Relative permeability calculations from pore size distribution data. Journal of Petroleum Technology 5, 71-78. 
[9] Coats, K.H., Smith, B.D., 1964. Dead-end pore volume and dispersion in porous media. SPE Journal 4, 78-84. doi:10.2118/647-PA.

[10] Cussler, E.L., 1997. Diffusion: Mass Transfer in Fluid Systems. Cambridge University Press.

[11] De Gennes, P., 1983. Hydrodynamic dispersion in unsaturated porous media. Journal of Fluid Mechanics 136, 189-200.

[12] De Smedt, F., Wauters, F., Sevilla, J., 1986. Study of tracer movement through unsaturated sand. Journal of Hydrology 85, 169181. URL: http://www.sciencedirect.com/science/article/pii/ 0022169486900831, doi:http://dx.doi.org/10.1016/0022-1694(86) 90083-1.

[13] De Smedt, F., Wierenga, P.J., 1979. Mass transfer in porous media with immobile water. Journal of Hydrology 41, 5967. URL: http://www.sciencedirect.com/science/article/pii/ 0022169479901057, doi:http://dx.doi.org/10.1016/0022-1694(79) 90105-7.

[14] De Smedt, F., Wierenga, P.J., 1984. Solute transfer through columns of glass beads. Water Resources Research 20, 225-232. URL: http://dx. doi.org/10.1029/WR020i002p00225, doi:10.1029/WR020i002p00225.

[15] Gaudet, J.P., Jégat, H., Vachaud, G., Wierenga, P.J., 1977. Solute transfer, with exchange between mobile and stagnant water, through unsaturated sand1. Soil Science Society of America Journal 41, 665-671. URL: http://dx.doi.org/10. 2136/sssaj1977.03615995004100040009x, doi:10.2136/sssaj1977. $03615995004100040009 x$.

[16] van Genuchten, M., Parker, J.C., 1984. Boundary conditions for displacement experiments through short laboratory soil columns. Soil Science Society of America Journal 48, 703-708.

[17] van Genuchten, M., Wierenga, P., 1976. Mass transfer studies in sorbing porous media. i. analytical solutions. Soil Science Society of America Journal , 473-480. 
[18] Godinez-Brizuela, O.E., Karadimitriou, N.K., Joekar-Niasar, V., Shore, C.A., Oostrom, M., 2017. Role of corner interfacial area in uniqueness of capillary pressure-saturation- interfacial area relation under transient conditions. Advances in Water Resources 107, 10 - 21.

[19] Griffioen, J.W., Barry, D.A., Parlange, J.Y., 1998. Interpretation of tworegion model parameters. Water Resources Research 34, 373-384. URL: http://dx.doi.org/10.1029/97WR02027, doi:10.1029/97WR02027.

[20] Haga, D., Niibori, Y., Chida, T., 1999. Hydrodynamic dispersion and mass transfer in unsaturated flow. Water Resources Research 35, 1065-1077. URL: http://dx.doi.org/10.1029/1998WR900111, doi:10.1029/1998WR900111.

[21] Harter, T., Yeh, T.C.J., 1996. Conditional stochastic analysis of solute transport in heterogeneous, variably saturated soils. Water Resources Research 32, 1597-1609.

[22] James, R.V., Rubin, J., 1986. Transport of chloride ion in a waterunsaturated soil exhibiting anion exclusion1. Soil Science Society of America Journal 50, 1142-1149. URL: http://dx.doi.org/10. 2136/sssaj1986.03615995005000050010x doi:10.2136/sssaj1986. $03615995005000050010 x$.

[23] Joekar-Niasar, V., Ataie-Ashtiani, B., 2009. Assessment of nitrate contamination in unsaturated zone of urban areas: The case study of tehran, iran. Environmental Geology 57, 1785-1798. URL: http://dx.doi. org/10.1007/s00254-008-1464-0, doi:10.1007/s00254-008-1464-0.

[24] Joekar-Niasar, V., Hassanizadeh, S.M., 2012. Analysis of fundamentals of two-phase flow in porous media using dynamic pore-network models: A review. Critical Reviews in Environmental Science and Technology 42, 1895-1976. URL: http://dx.doi.org/10.1080/10643389.2011. 574101, doi:10.1080/10643389.2011.574101.

[25] Joekar-Niasar, V., Mahani, H., 2016. Nonmonotonic pressure field induced by ionic diffusion in charged thin films. Industrial \& Engineering Chemistry Research 55, 6227-6235.

[26] Karadimitriou, N.K., Joekar-Niasar, V., Babaei, M., Shore, C.A., 2016. Critical role of the immobile zone in non-fickian two-phase transport: 
A new paradigm. Environmental Science and Technology 50, 43844392. URL: http://dx.doi.org/10.1021/acs.est.5b05947, doi:10. 1021/acs.est.5b05947.

[27] Karadimitriou, N.K., Joekar-Niasar, V., Godinez-Brizuela, O., 2017. Hydro-dynamic solute transport under two-phase flow conditions. Scientific Reports 7, 6624.

[28] Krupp, H.K., Elrick, D.E., 1968. Miscible displacement in an unsaturated glass bead medium. Water Resources Research 4, 809-815. URL: http://dx.doi.org/10.1029/WR004i004p00809, doi:10.1029/ WR004i004p00809.

[29] Li, M., Vashaee, S., Romero-Zerón, L., Marica, F., Balcom, B.J., 2017. A magnetic resonance study of low salinity waterflooding for enhanced oil recovery. Energy \& Fuels 31, 10802-10811.

[30] Maciejewski, S., 1993. Numerical and experimental study of solute transport in unsaturated soils. Journal of Contaminant Hydrology 14, 193206. URL: http://www.sciencedirect.com/science/article/pii/ 016977229390024M, doi:http://dx.doi.org/10.1016/0169-7722(93) 90024-M.

[31] Mahani, H., Berg, S., Ilic, D., Bartels, W.B., Joekar-Niasar, V., 2015. Kinetics of low-salinity-flooding effect. SPE Journal 20, 8-20. doi:10. 2118/165255-PA.

[32] Maraqa, M.A., B. Wallace, R., C. Voice, T., 1997. Effects of degree of water saturation on dispersivity and immobile water in sandy soil columns. Journal of Contaminant Hydrology 25, 199-218. URL: http://www.sciencedirect.com/science/ article/pii/S0169772296000320, doi/http://dx.doi.org/10.1016/ S0169-7722(96)00032-0.

[33] Morrow, N., Buckley, J., 2011. Improved oil recovery by low-salinity waterflooding. Journal of Petroleum Technology 63, 106 - 112.

[34] Ogata, A., Banks, R.B., 1961. A solution of the differential equation of longitudinal dispersion in porous media: fluid movement in earth materials. US Government Printing Office. 
[35] Padilla, I.Y., Yeh, T.C.J., Conklin, M.H., 1999. The effect of water content on solute transport in unsaturated porous media. Water Resources Research 35, 3303-3313. URL: http://dx.doi.org/10.1029/ 1999WR900171, doi:10.1029/1999WR900171.

[36] Roth, K., Hammel, K., 1996. Transport of conservative chemical through an unsaturated two-dimensional miller-similar medium with steady state flow. Water Resources Research 32, 1653-1663. URL: http://dx.doi. org/10.1029/96WR00756, doi:10.1029/96WR00756.

[37] Sahimi, M., Heiba, A.A., Davis, H.T., Scriven, L., 1986. Dispersion in flow through porous media - ii. two-phase flow. Chemical Engineering Science 41, $2123-2136$.

[38] Scheidegger, A.E., 1961. General theory of dispersion in porous media. Journal of Geophysical Research 66, 3273-3278. URL: http://dx.doi. org/10.1029/JZ066i010p03273, doi:10.1029/JZ066i010p03273.

[39] Schulin, R., Wierenga, P.J., Flühler, H., Leuenberger, J., 1987. Solute transport through a stony soil1. Soil Science Society of America Journal 51, 36-42. URL: http://dx.doi.org/10. 2136/sssaj1987.03615995005100010007x doi:10.2136/sssaj1987. $03615995005100010007 \mathrm{x}$.

[40] Springer, J., Urban, K., Kissling, K., Schütz, S., Piesche, M., Jasak, H., 2010. A coupled pressure based solution algorithm based on the volume of fluid approach for two or more immiscible fluids, in: OpenFOAM Workshop, Gothenburg, Sweden.

[41] Toride, N., Inoue, M., Leij, F.J., 2003. Hydrodynamic dispersion in an unsaturated dune sand. Soil Science Society of America Journal 67, 703-712. URL: http://dx.doi.org/10.2136/sssaj2003.0703, doi:10.2136/sssaj2003.0703.

[42] Wei, B., Wu, R., Lu, L., Ning, X., Xu, X., Wood, C., Yang, Y., 2017. Influence of individual ions on oil/brine/rock interfacial interactions and oil--water flow behaviors in porous media. Energy \& Fuels 31, 1203512045 . 
[43] Weller, H.G., Tabor, G., Jasak, H., Fureby, C., 1998. A tensorial approach to computational continuum mechanics using object-oriented techniques. Computers in Physics 12, 620-631.

[44] Yule, D.F., Gardner, W.R., 1978. Longitudinal and transverse dispersion coefficients in unsaturated plainfield sand. Water Resources Research 14, 582-588. URL: http://dx.doi.org/10.1029/WR014i004p00582, doi:10.1029/WR014i004p00582. 
Supplementary Information

\title{
Pore-scale insights into transport and mixing in steady-state two-phase flow in porous media
}

\author{
Rimsha Aziz ${ }^{1}$, Vahid Joekar-Niasar, ${ }^{1, *}$ Pedro Martinez-Ferrer ${ }^{2}$ \\ ${ }^{1}$ School of Chemical Engineering and Analytical Science, University of \\ Manchester, Manchester, M13 9PL, UK \\ ${ }^{2}$ Centre for Mathematical Modelling and Flow Analysis, Chester Street, \\ Manchester, M1 5GD, United Kingdom
}

\section{Simulation details}

The numerical domain is shown in Figure S1. The mean grain size is $50 \mu m$ and the porosity is $52 \%$. The circle packing was generated such that no dead-end pore to be created. the top and bottom boundaries are no-flow boundaries and the inlet and outlet are situated in the left and right, respectively. The porosity analysis has been done to determine the REA size. The selected domain size is roughly 2 REA at each direction (4REAs in total). Only steady-state primary drainage simulations were performed to create the saturation topology.

We performed the grid convergence analysis in a sub-section of the original domain. The grid convergence was done based on the mass balance analysis in a two-phase flow. For different grid resolutions we simulated two-phase flow at the same injection rate on the same domain using the same numerical schemes. We quantified the change of saturation from the simulation results before the breakthrough, referred to as $E_{i}$. Also, since the boundary condition was fixed flow rate, we easily calculated the change of saturation before breakthrough based on the injection rate, referred to as $E$. The relative error (\%) at each

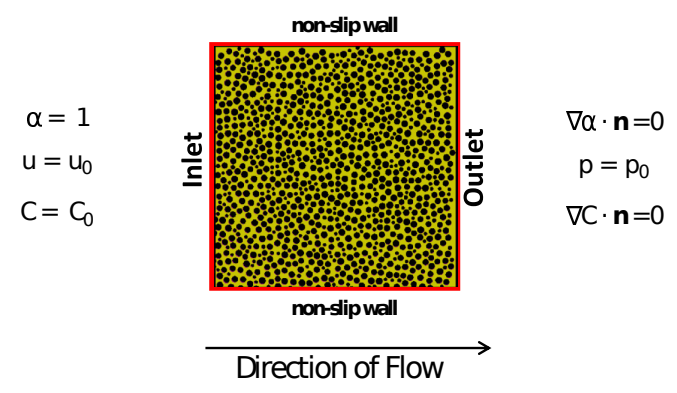

Figure S1: 2D demonstration of the numerical domain, $\alpha$ and $\mathrm{C}$ are initially set to zero in internal domain. 


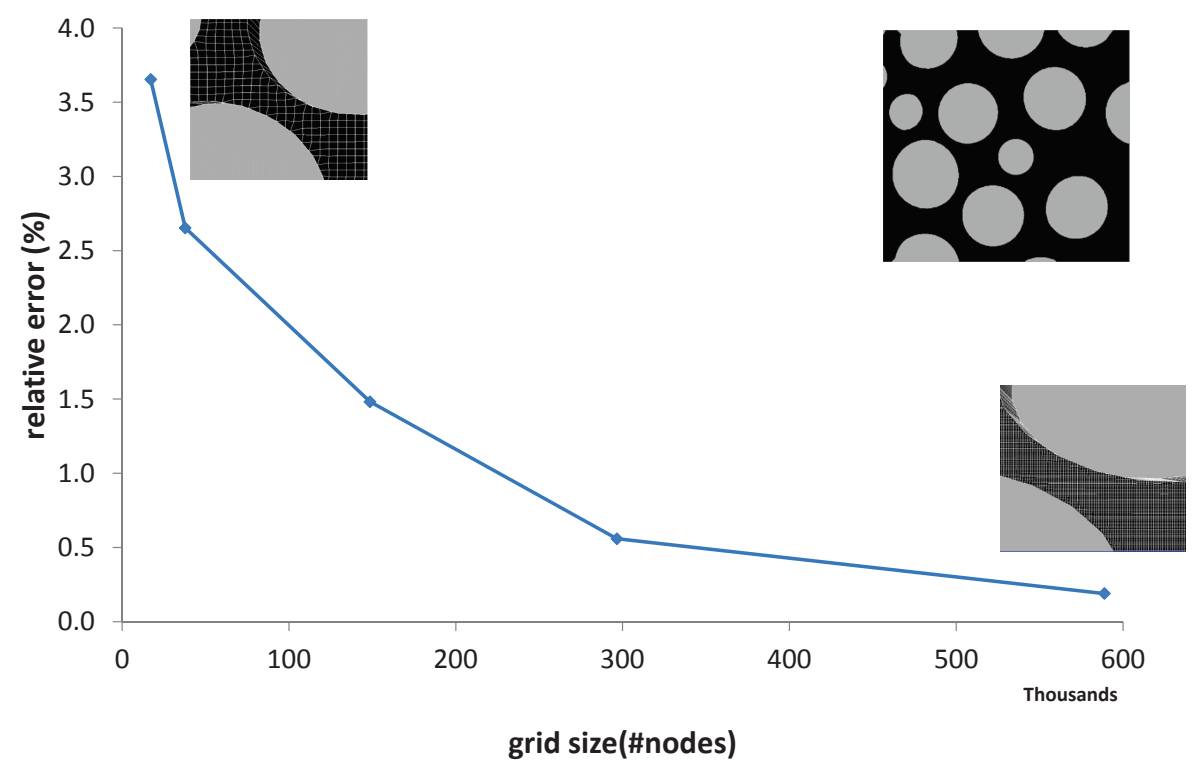

Figure S2: Grid convergence analysis based on the mass balance in a two-phase flow before breakthrough.

simulation case was calculated as $\operatorname{ABS}\left(E-E_{i}\right) / E \times 100$. Results are shown in Figure S2,

Based on the grid convergence analysis shown in Figure S2 the error minimized after 300,000 grid size. Therefore, we selected this mesh size to perform all our simulations. Maintaining the same grid resolution for the whole domain $(\delta x / L \approx 0.0003)$, our final mesh contained 3.6 million cells. This resolution provided at least 15 cells in the narrowest pore throat which ensured the sharp changes in volume fraction were captured accurately.

Curve fitting to estimate the dispersion coefficient $D$ and effective pore velocity $v$

We established steady-state two-phase saturations, ranging between $0.34-1$ to be used for transport simulations. We then calculated the resident concentration in the flowing regions and produced the time series of concentration profiles at different Péclet numbers and saturations, which followed the Fickian transport.

To obtain the dispersion coefficient and the effective pore velocity, the resident concentration profiles in the flowing regions were fitted using Equation 7. The curve fitting results were excellent with RMSE ranging from $10^{-7}-10^{-5}$. Two examples of the curve fitting at two different saturation at Pećlet number of 700 are shown in Figure S3 

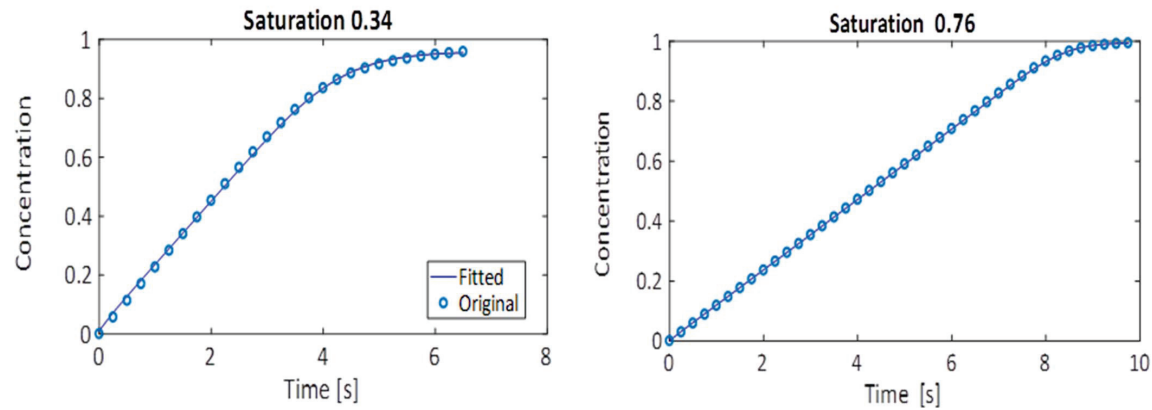

Figure S3: Curve fitting of the resident concentration in the flowing region at saturations of 0.34 and 0.76 at Pećlet number of 700 .

Dead-End Pore (DEP) and Mobile-Immobile (MIM) models

The dead-end pore (DEP) [1] and the mobile-immobile model (MIM) [2] are the two notable Darcy-scale models to estimate transport parameters as well as stagnant saturation in two-phase flow. The differences between the two models are highlighted below.

The DEP model uses the following equations to simulate transport in twophase flow.

$$
\begin{gathered}
S_{f w} \frac{\partial C}{\partial t}+S_{s w} \frac{\partial C_{s w}}{\partial t}=D \frac{\partial^{2} C}{\partial x^{2}}-v \frac{\partial C}{\partial x} \\
S_{s w} \frac{\partial C}{\partial t}=\gamma_{d e p}\left(C-C_{s w}\right)
\end{gathered}
$$

In Equation S1, $S_{f w}$ is saturation of the flowing water, $C$ is the total concentration in water, $t$ is time, $S_{s w}$ is saturation of the stagnant water, $C_{s w}$ is the concentration in the stagnant water, $D$ is the longitudinal dispersion coefficient, $v$ is the effective pore velocity and $x$ is the direction parallel to flow. To account for mixing, $\gamma_{d e p}$ (exchange rate coefficient) was introduced. $S_{s w}, v$, $\mathrm{D}$ and $\gamma_{d e p}$ were estimated using curve fitting to match the experimental data. Note that the exchange rate is introduced between the total concentration and the stagnant concentration.

In the MIM model, the authors improved equations of the DEP model by decoupling the water domain to the immobile (stagnant) and mobile (flowing) regions. They clearly distinguished the concentration in the flowing $\left(C_{f w}\right)$ and stagnant regions $\left(C_{s w}\right)$. The equations for MIM are given as follows:

$$
\begin{aligned}
S_{f w} \frac{\partial C_{f w}}{\partial t}+S_{s w} \frac{\partial C_{s w}}{\partial t} & =S_{f w} D \frac{\partial^{2} C_{f w}}{\partial x^{2}}-v S_{f w} \frac{\partial C_{f w}}{\partial x} \\
S_{s w} \varphi \frac{\partial C_{s w}}{\partial t} & =\gamma\left(C_{f w}-C_{s w}\right)
\end{aligned}
$$


In literature, either the resident or breakthrough concentration profiles were fitted using the MIM model to estimate $S_{s w}, \gamma, v$, and $D$ and none of these parameters were directly estimated for laboratorial experiments.

-There are two main drawbacks in the DEP and MIM models, which are explained here. If the flow rate is at the same scale of diffusion in experimental data (i.e. $P_{e}$ is small), the curve fitting will result a small dispersion coefficient and smaller stagnant saturation. This implies that for the same saturation topology, $P_{e}$ is controlling the stagnant saturation and not the saturation topology. We interpret this as a main flaw in the MIM model, as the stagnant saturation should not change as long as the saturation topology does not change. Also, the MIM model does not have any link with the two-phase flow properties and it does not explain how the transport properties are changing as a consequence of two-phase flow properties. Our results clearly highlight the first drawback that the stagnant saturation is a function of saturation topology and as long as the saturation does not change the stagnant saturation should not change with $P_{e}$ number. Regarding the second drawback, we have shown that there is a correlation between the relative permeability curves and the stagnant saturation.

\section{References}

[1] Coats, K.H., Smith, B.D., 1964. Dead-end pore volume and dispersion in porous media. SPE Journal 4, 78-84. doi 10.2118/647-PA.

[2] van Genuchten, M., Wierenga, P., 1976. Mass transfer studies in sorbing porous media. i. analytical solutions. Soil Science Society of America Journal , 473-480. 